\title{
¿ACTIVIDAD LIBERAL O LIBERTINAJE? LA PRÁCTICA LABORAL EN LA ARQUEOLOGÍA DE CONTRATO EN URUGUAY.
}

\author{
Irina Capdepont Caffa
}

\begin{abstract}
Resumen: El desarrollo económico y los procesos de modernización gestados en Uruguay, generan transformaciones afectando al patrimonio arqueológico mediante la ejecución de obras. El aumento de estas, junto con la Ley de Impacto Ambiental forjó la ejecución de estudios de impacto arqueológico. Ello genero un crecimiento en el campo laboral que no ha sido acompañado de un cambio legal, administrativo y académico. La problemática originada por todo ello hacen abordar y reflexionar sobre la situación actual del accionar arqueológico, los procedimientos metodológicos y éticos y el rol de los organismos fiscalizadores en el contexto de la Arqueología de Contrato o Arqueología Aplicada.
\end{abstract}

Palabras Claves: Impacto Arqueológico, Campo Laboral

\section{I ntroducción}

Con el aumento de las obras públicas y privadas y la existencia de la Ley de Medio Ambiente 16.464, la Dirección Nacional de Medio Ambiente contempla el relevamiento y rescate de sitios de valor cultural, situación que debe reflejarse en las Evaluaciones de Impacto Ambiental. La Ley de Medio Ambiente, promulgada en 1994, cuanta con 18 Artículos y un Decreto Reglamentario, de los cuales se desprenden y enmarcan las ejecuciones de los Estudios de Impacto Arqueológico:

Art. 2o de la Ley de Medio Ambiente 16.464/ 94.- Se considera impacto ambiental negativo o nocivo toda

MUNHINA Ministerio de Educación y Cultura, Montevideo - Uruguay / FCS - Universidad del Centro de la Provincia de Buenos. Aires.iracap@yahoo.com.ar alteración de las propiedades físicas, químicas o biológicas del medio ambiente causada por cualquier forma de materia o energía resultante de las actividades humanas que directa o indirectamente perjudiquen o dañen: La salud, seguridad o calidad de vida de la población. Las condiciones estéticas, culturales o sanitarias del medio. La configuración, calidad y diversidad de los recursos naturales (Diario Oficial № 23977 1994).

Art. 12 del Decreto Reglamentario 435/94.- El documento que recoja los resultados del Estudio de Impacto Ambiental, deberá contener, como mínimo, las características del ambiente receptor, en la que se describirán las características del entorno, se evaluarán las afectaciones ya existentes y se identificarán las áreas sensibles o de riesgo; todo ello en tres aspectos: 
a) Ambiente físico: agua, suelo, paisaje, etc.

b) Ambiente biótico: fauna, flora, biota acuática, etc.

c) Ambiente antrópico: población, actividades, usos del suelo, sitios de interés histórico y cultural. (Diario Oficial № 24/145 1994).

La Ley de Medio Ambiente y el Decreto Reglamentario han contribuido a generar un gran crecimiento en lo referente al: campo laboral del arqueólogo, al conocimiento de nuevas áreas y a la profundización de información en áreas ya conocidas. Este crecimiento no ha sido acompañado por un cambio específico en la administración pública ni en el accionar de los profesionales involucrados. Lo expuesto lleva a la necesaria reflexión crítica sobre la situación actual del campo laboral en arqueología, haciendo especial hincapié en los procedimientos éticos y metodológicos, en el rol de los organismos fiscalizadores (Comisión del Patrimonio Cultural de la Nación-CPCN, Dirección Nacional de Medio Ambiente-DINAMA, Dirección Nacional de Minería y Geología-DINAMIGE) y de los profesionales que actúa sobre el Patrimonio Arqueológico.

\section{Marco Conceptual}

Para comenzar a plantear algunos de los puntos de este trabajo, parece conveniente primeramente mencionar los principios de los que se parte para desarrollar el tema de la Arqueología de Contrato llevada a cavo a través de los Estudios de impacto arqueológico (El Arq de aquí en adelante). Algunos de estos principios son:

- Entender al Patrimonio Arqueológico comprendido en tres dimensiones: como objeto real, como documento de las sociedades pasadas y como recurso de las sociedades actuales (sensus Criado 1996). Ello implica tener que gestionarlo de forma integral: inventariarlo, describirlo, analizarlo, valorarlo y difundirlo (Criado 2003).
- El adecuado tratamiento y manejo del patrimonio arqueológico (Gestión I ntegral sensus Amado et al. 2002) es compatible con el desarrollo económico del país. La existencia de un sitio arqueológico en un área determinada no imposibilita habitualmente el desarrollo de otras actividades.

- Los El Arq frecuentemente se enmarcan dentro de la práctica liberal de la profesión. Con ello se entiende el ejercicio de una actividad intelectual profesional sin vínculos estables con personas o instituciones para la prestación de servicios. Las relaciones laborales cesan al concluirse el acto profesional y donde la obligación asumida por parte del profesional tiende a obtener un determinado resultado, en un tiempo y a un costo pactado entre los involucrados. Ello implica también la responsabilidad frente a los actos realizados en un EIArq.

- Se concibe al ElArq como un proceso continuo en el que interrelacionan arqueólogos y empresas, y como una parte más del proyecto de construcción durante su fase de diseño y planificación, lo que evita los problemas de mayor gravedad en momentos ulteriores de la ejecución del proyecto (Criado et al. 2004).

- Entre los objetivos de un El Arq se deben encontrar:

a - Diagnosticar, prevenir, mitigar, corregir y/o compensar el impacto sobre el Patrimonio Arqueológico, revirtiendo los efectos negativos mediante la producción de conocimiento de relevancia científica y social.

b - Dar soluciones a problemas específicos relacionados con proyectos de desarrollo o actividades económicas.

c - Gestionar de forma Integral el Patrimonio Cultural y que ese Patrimonio no sufra significativas alteraciones a través del desarrollo de diferentes emprendimientos económicos y procesos de modernización. 
d - Valorar los impactos y generar propuestas de mitigación.

- Considerar las diferencias y similitudes existentes entre la Arqueología Aplicada o de Contrato y la Arqueología Académica, ya que si bien comparten la rigurosidad metodológica y la generación conocimientos, difieren en varios puntos (Tabla 1).

- Se entiende a la Arqueología Aplicada como una Arqueología que permite pensar la Arqueología, pensar la relación que debe establecerse entre ésta y la Sociedad, que genera nuevos valores, que contribuye a erradicar unos y a transformar otros. Una Arqueología Aplicada es entonces una Arqueología desde la reflexión y para la acción, planteada en el contexto actual, en donde tienen cabida nuevas necesidades y viejos problemas, a los que podemos intentar dar respuesta manteniendo vigente el componente epistemológico y axiológico que debe acompañar toda disciplina (Barreiro 2005:22).

\section{Tabla 1}

\section{Arqueología Aplicada o de Contrato}

Investigaciones en Obras Públicas/Privadas

\section{Arqueología Académica}

nvestigación en General
Arqueología como disciplina para la gestión del patrimonio arqueológico (Barreiro 2005:22).

Tiempos Cortos para desarrollar las actividades.

Costos económicos altos.

Responsabilidades de todos.

Genera un ámbito laboral.

Es una necesidad social derivada del desarrollo de las políticas de planeamiento 0 de infraestructuras (Cabrejas 2004).

La Arqueología como un saber-hacer-cosas (Criado 1996).

Resuelve problemas prácticos, se orienta hacia su resolución y se materializa en una oferta de servicios concreta (Criado 1996).
Arqueología entendida como disciplina independiente del contexto social en que se genera o sólo unida a él por el cordón umbilical de las aulas universitarias (Barreriro 2005:22).

Tiempos Largos para desarrollar las actividades.

Costos relativos.

Responsabilidades de algunos.

Genera preparación académica.

Necesidad para la generación de conocimiento científico.

La Arqueología como saber académico.

Resuelve problemas de conocimiento particulares y se materializa en artículos científicos.

Rigurosidad metodológica.

Generación de Conocimientos del área estudiada.

En la tabla se exponen de forma esquemática algunas de las similitudes y diferencias entre la Arqueología Aplicada a través de la investigación en obras públicas/privadas y la Arqueología Académica. 
- Los ElArq generan el crecimiento del campo laboral del arqueólogo, del conocimiento de nuevas áreas y de la profundización de información de áreas ya conocidas.

\section{Situación actual}

El Patrimonio de Uruguay no posee aún el amparo legal, administrativo ni científico necesario para que se pueda Gestionar de forma integral (inventariar, describir, analizar, valorar y difundir). Una de las herramientas indispensables y básicas para gestionar el Patrimonio son los inventarios. En este sentido, Uruguay cuenta con la Ley de Patrimonio 14.040 de 1971 que establece, entre otras cosas, la necesidad de realizar y publicar el inventario del patrimonio histórico, artístico y cultural de la nación (Diario Oficial № 18667 1971). Interesa resaltar como esta conformado ese inventario: el mismo cuenta con $76 \%$ de bienes de la época histórica y colonial, $23 \%$ de la época histórica moderna y $1 \%$ de bienes prehistóricos compuestos por
2 colecciones de objetos y 13 sitios con arte rupestre (Registro de Monumentos Históricos Nacionales 2006) (Gráfico 1).

Evidentemente el inventario presenta un vacio en la representación de la prehistoria del territorio producto de la época en el que el mismo se realizó (amparado en la historiografía oficialista en donde los bienes patrimoniales debían corresponderse con la identidad colonial blanca). En dicho contexto, mediados del S. XIX, la nación va tomando forma mediante la adopción de estrategias y valores provenientes de Occidente (Verdesio 2004). Ese vacio en el inventario, se correspondería a un olvido del pasado, que bien podría comenzar a completarse a través, entre otras, de la información generada por los ElArq y el interés de la administración y los profesionales involucrados en los mismos.

Con la finalidad de observar el estado de la práctica liberal en Uruguay y comenzar a inventariar información arqueológica, se hizo una revisión de informes de El Arq que se encontraban en el Departamento de Arqueología de la Comisión del Patrimonio Cultural de la Nación (CPCN) - Ministerio de Educación y Cultura.

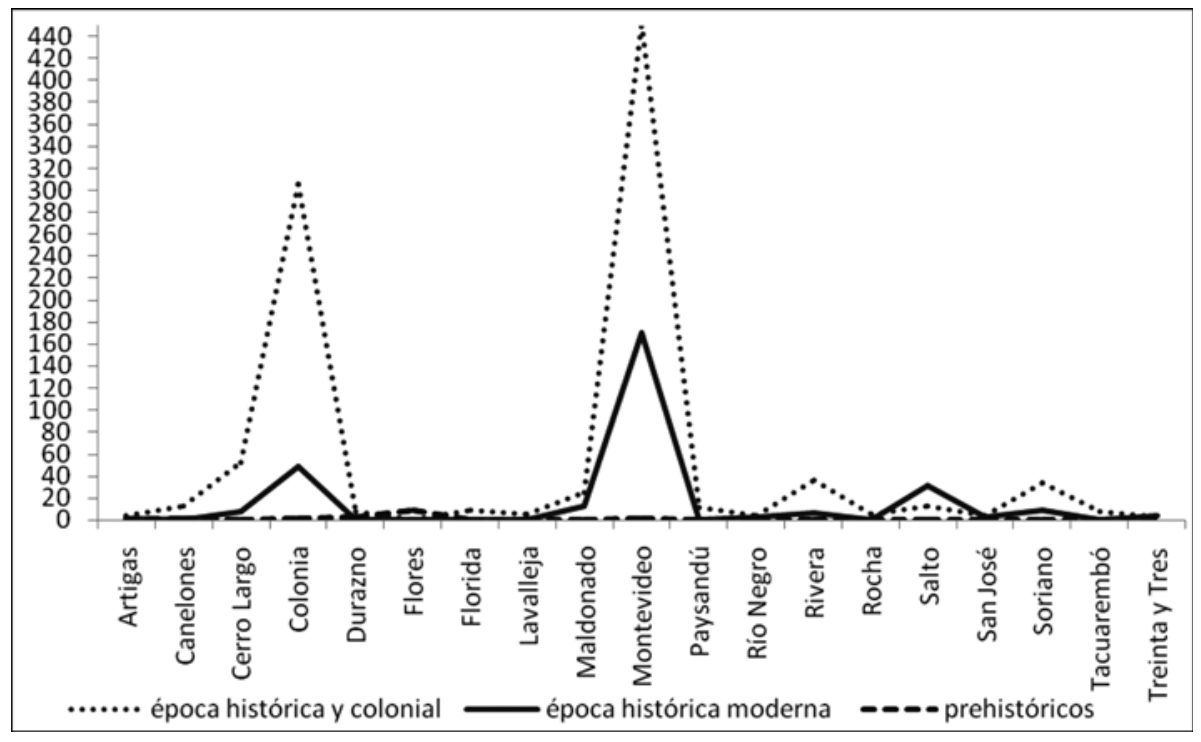

Grafico 1. En el gráfico se expresa por Departamento el número de Monumentos Históricos Nacionales para el territorio uruguayo. 
En los gráficos 2 y 3 se puede observar la evolución desde 1996 de las actuaciones de El Arq llevados a cabo en Uruguay que han pasado por la CPCN. En los mismo se evidencia un nítido incremente a partir de fines de 1999 y principios del 2000 en el que se registra el aumento de las intervenciones sobre el territorio, el número de arqueólogos participantes y la cantidad de ElArq realizados. Asimismo, de acuerdo a los datos manejados hasta ese momento, las obras mayormente representadas son las

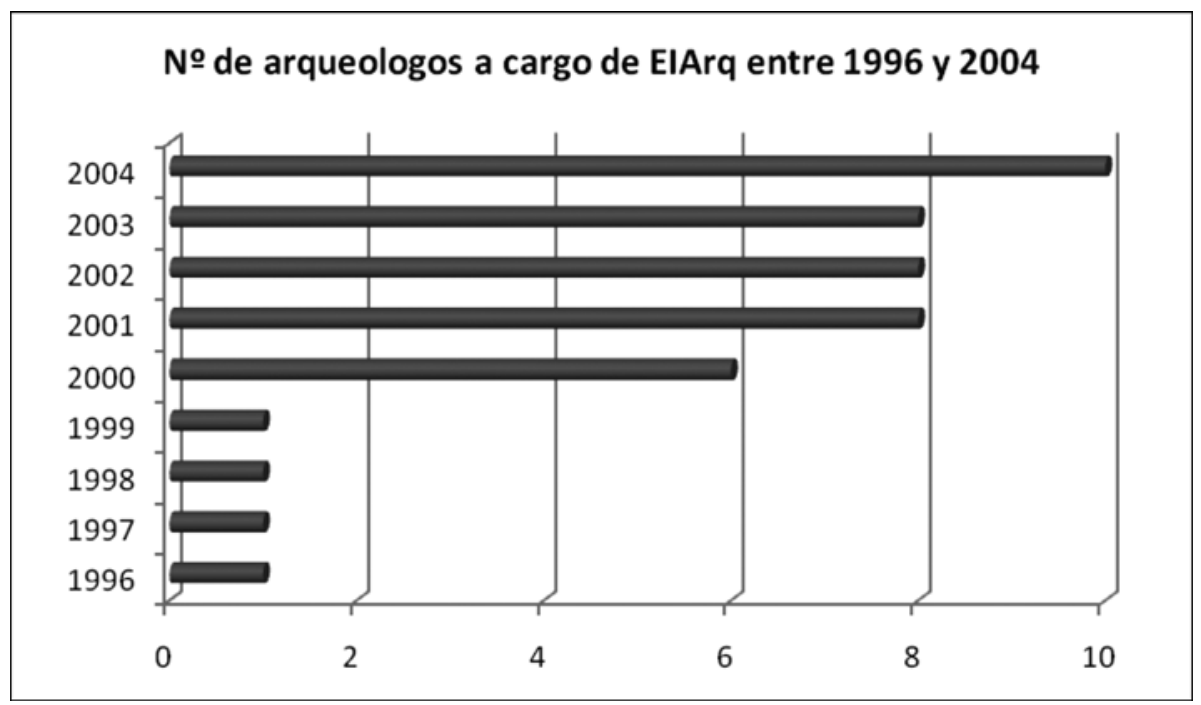

Grafico 2. En este gráfico se expone el número total de arqueólogos que ha desarrollado entre 1996 y el 2004 EIArq.

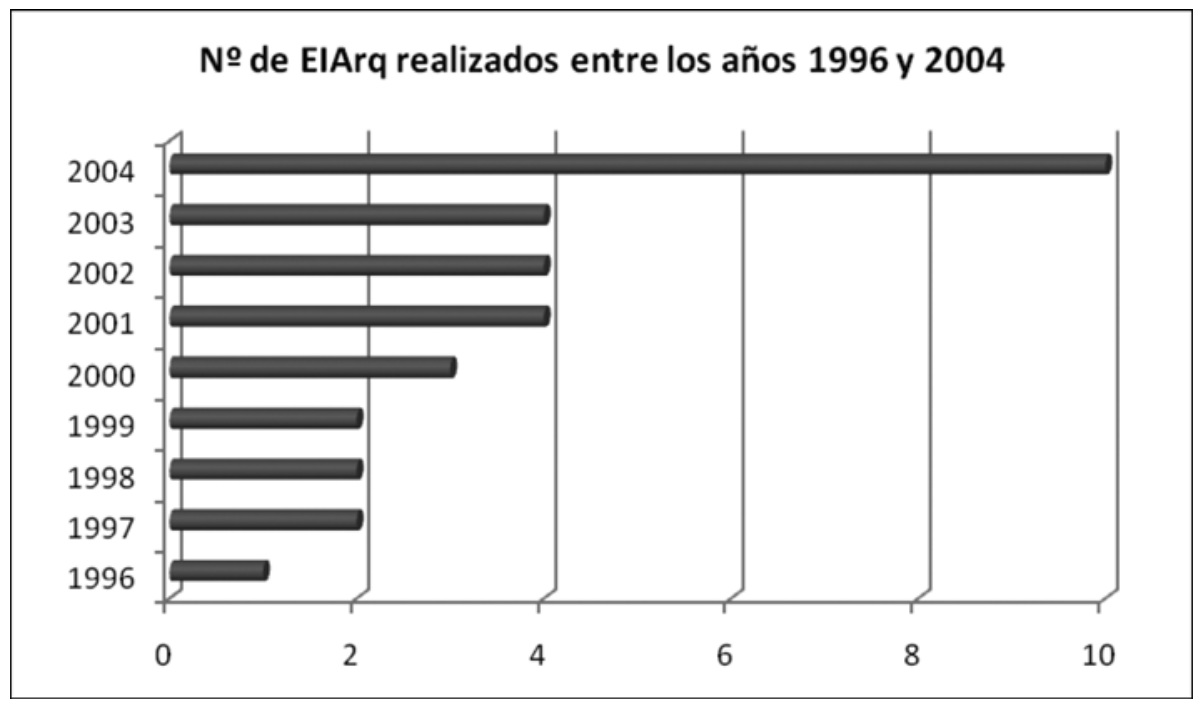

Grafico 3. Se expone en el gráfico la cantidad de ElArq desarrollados entre los años 1996 y 2004 en territorio uruguayo. 
correspondientes a la extracción de minerales en los Departamentos de San J osé y Colonia (Grafico 4 y 5 ). No obstante, en los últimos años las actividades mineras se han incrementado en el departamento de Artigas.
En los relevamientos realizados se evidencia, que las evaluaciones de impacto arqueológico carecen de un modelo recomendado por instancias profesionales y administrativas, generando resultados muy disímiles con apenas puntos de coincidencia. Se ha

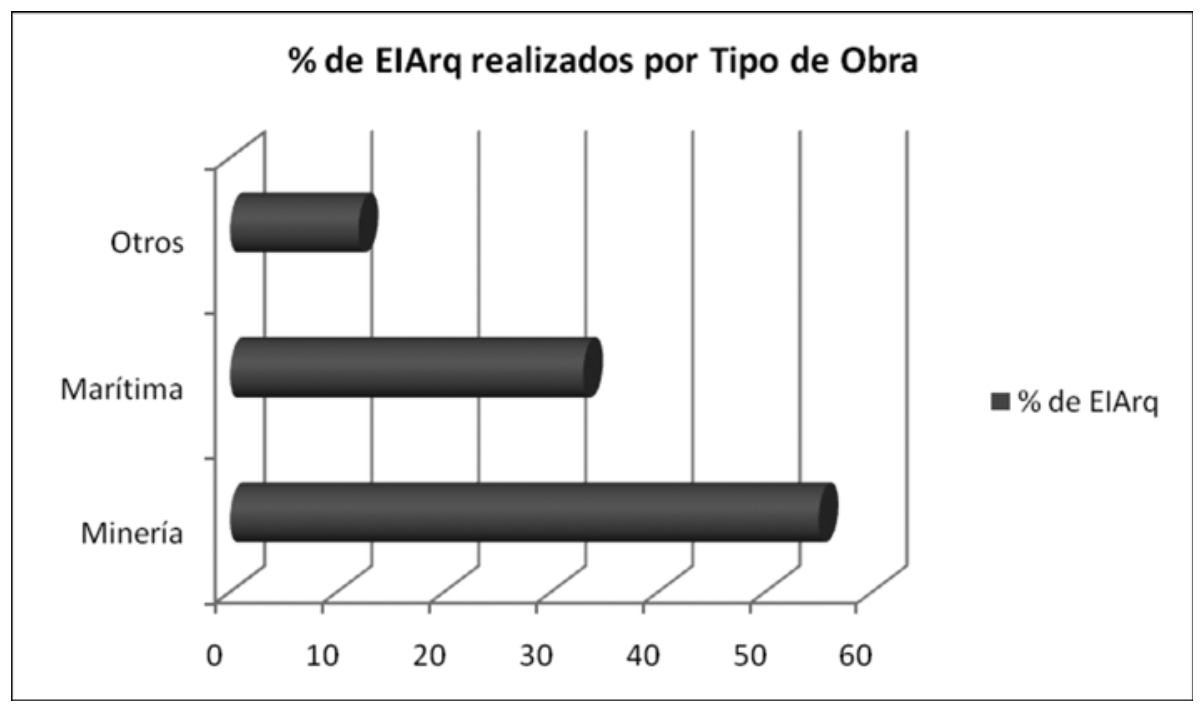

Grafico 4. Se observan los tipos y cantidad de obras en las cuales se han centrado los ElArq entre el año 1996 y 2004.

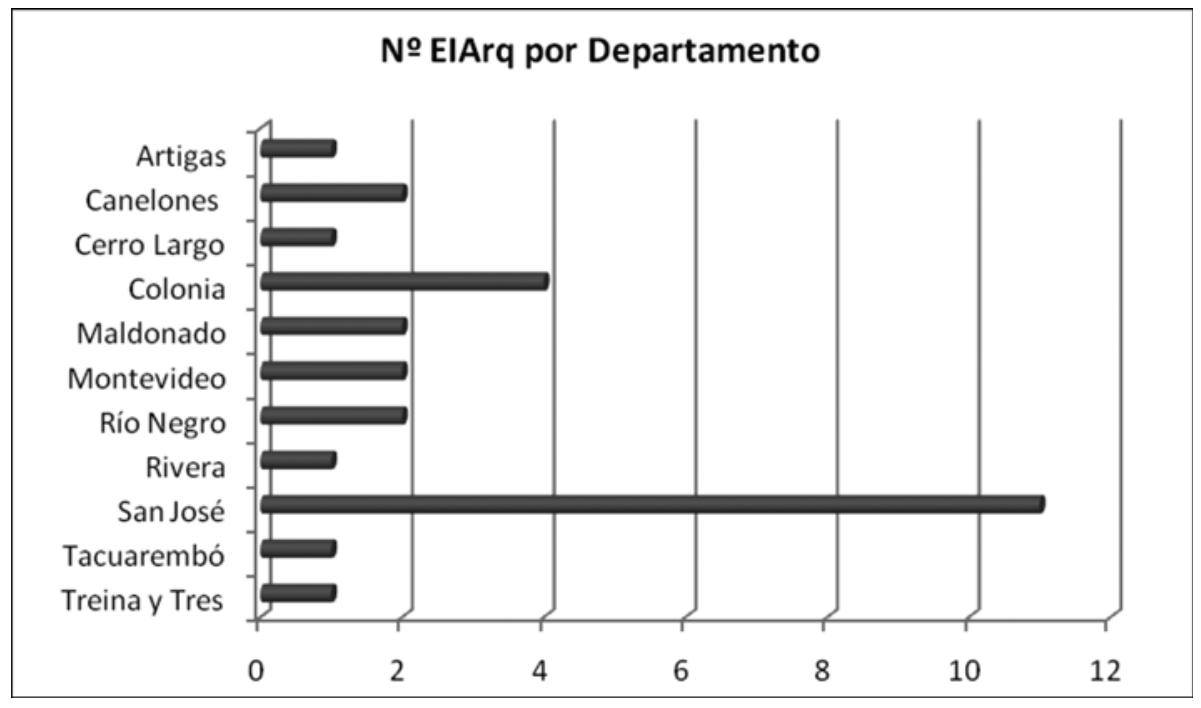

Grafico 5. Se expresa por Departamento el total de ElArq realizados en el correr de ocho años. 
observado que los criterios de evaluación de los informes presentados, así como los criterios de la administración para abordar los mismos, son muy dispares. Por ejemplo, en la Tabla 2 se puede observar resumidamente datos de algunos informes en los que se presentan evidencias de materiales arqueológicos y no se hace mención de sus características, lugar donde se encuentran depositados, ni recomendaciones mínimas (medidas correctoras). De forma contraria, en otros ElArq se presentan las características de la cultura material hallada y una serie de recomendaciones. Esta disparidad genera problemas prácticos importantes y redundan en contra de la calidad de los trabajos en directo perjuicio del patrimonio cultural.

Como se observa en la Tabla 2, los informes relevados involucran el período comprendido entre 1996 y principios del 2005; desde la fecha aún no se ha generado el interés, esfuerzo y compromiso conjunto de los profesionales mediante un consenso en lo referente a modelos de trabajo, metodologías de valoración, herramientas practicas, qué hacer y cómo hacerlo. Es así que se suscitan graves problemas (ver Expedientes 0557/01, 0742/02, 0609/05 y 0122/ 06, 0123/06 entre otros) como ser:

- Informes de Evaluación de Impacto Arqueológico sin las mínimas propuestas de medidas correctoras,

- Informes que pudieron realizarse sin que necesariamente el arqueólogo tuviera que trasladarse a campo,

-I nformes realizados y evaluados por parte de un mimo individuo (juez y parte),

- Controles realizados por la CPCN sin aviso previo a los responsables de los ElArq generando la inspección a lugares equivocados, entre otros.

Las problemáticas enunciadas se han credo debido a la ausencia de estándares que establezcan al menos algunos lineamientos metodológicos y técnicos para los EIArq, a la ausencia de un control de gestión efectivo e igual para todos los El Arq, al uso de criterios dispares en las evaluación de los ElArq y las irregularidades generadas por algunos individuos en el manejo de asuntos de injerencia exclusiva de la CPCN y el profesional actuante. Asimismo, el mal manejo conceptual de la actividad arqueológica en un estudio de impacto lleva a la errónea concepción de que un sitio arqueológico es un obstáculo para el desarrollo de actividades productivas. Esta concepción ha generado la errónea conclusión de que la existencia de un sitio arqueológico en un área específica imposibilita el desarrollo de cualquier actividad productiva. No obstante, existen profesionales que consideran que el adecuado tratamiento y manejo del recurso arqueológico es compatible con el desarrollo económico del país.

La inexistencia de asociaciones profesionales fuertes y protocolos para abordar los ElArq, entre otros factores, hace que algunos sujetos con su accionar generen perjuicios importantes sobre el patrimonio cultural, el organismo fiscalizador y el nuevo campo laboral arqueológico. Conjuntamente con ello, al no poseer el organismo fiscalizador bases claras e iguales para tratar todos los ElArq el funcionamiento liberal del ejercicio se convirtió ya en libertinaje, es decir en la perdida total de responsabilidad frente a los actos realizados.

Cabe mencionar, que gran parte de la problemática enunciada también se debe a que muchos arqueólogos de los que hoy se dedican a estudios de impacto no se han formado en dicha actividad, ni han procurado actualizarse mediante los cursos impartidos sobre el tema en la Facultad de Humanidades y Ciencias de la Educación. Este quehacer arqueológico ha sido tomado como mero generador de capital sin considerar que desde hace años se imparten en varias partes del mundo cursos de grado y postgrado específicos a nivel teórico-metodológico para abordar estudios de esa naturaleza.

\section{Propuesta}

De acuerdo a las problemáticas planteadas y considerando que Uruguay no 


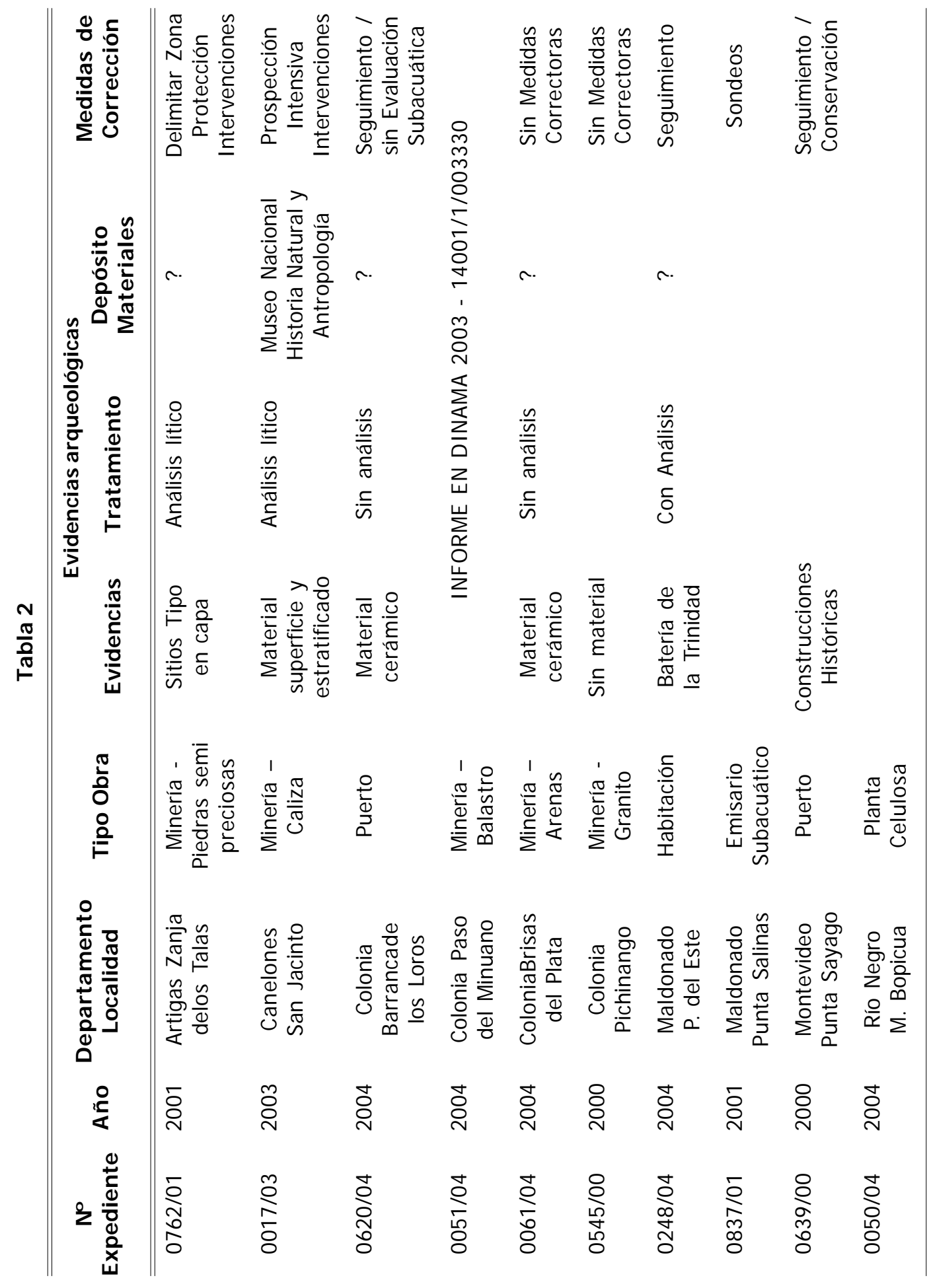




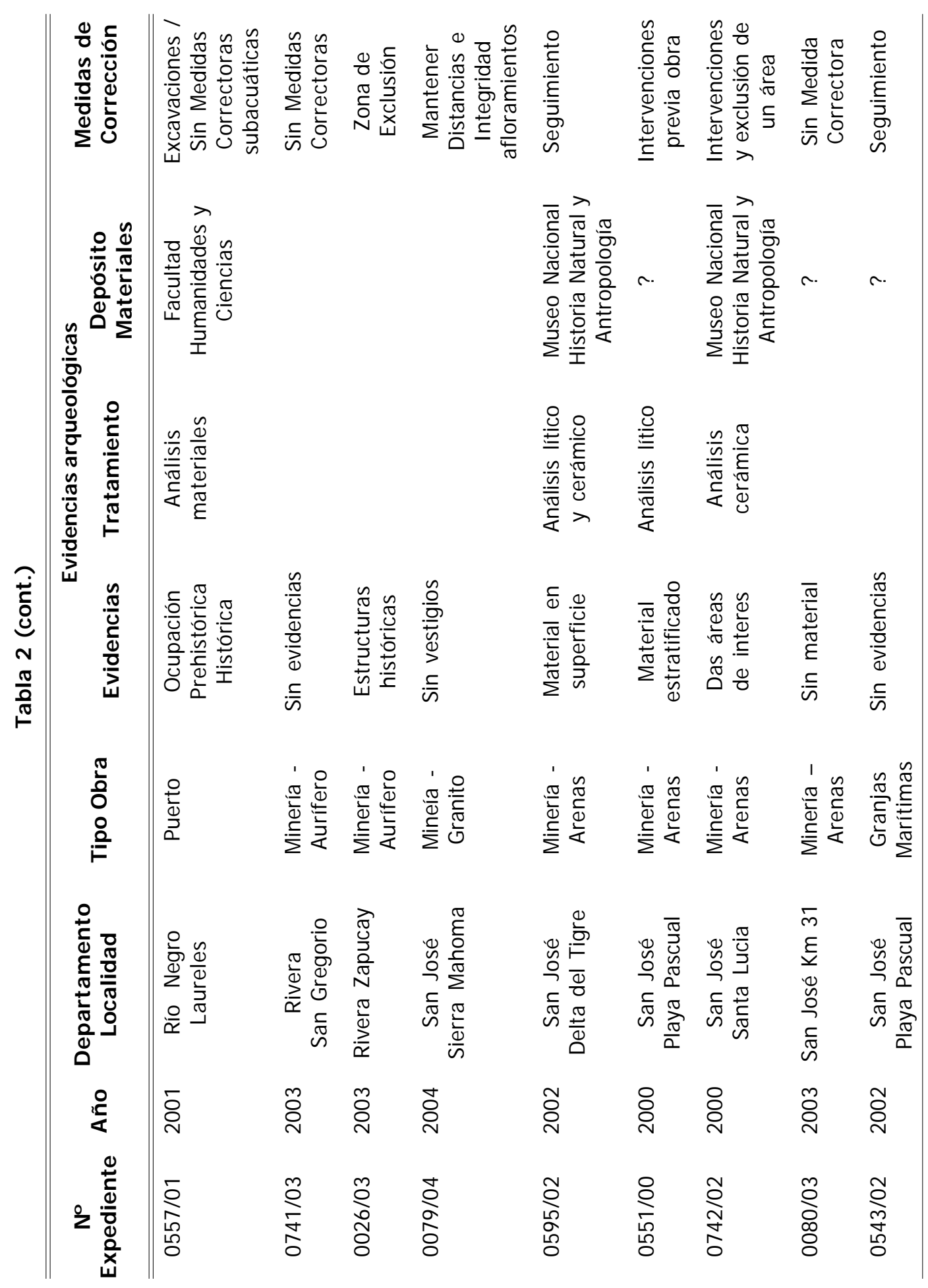




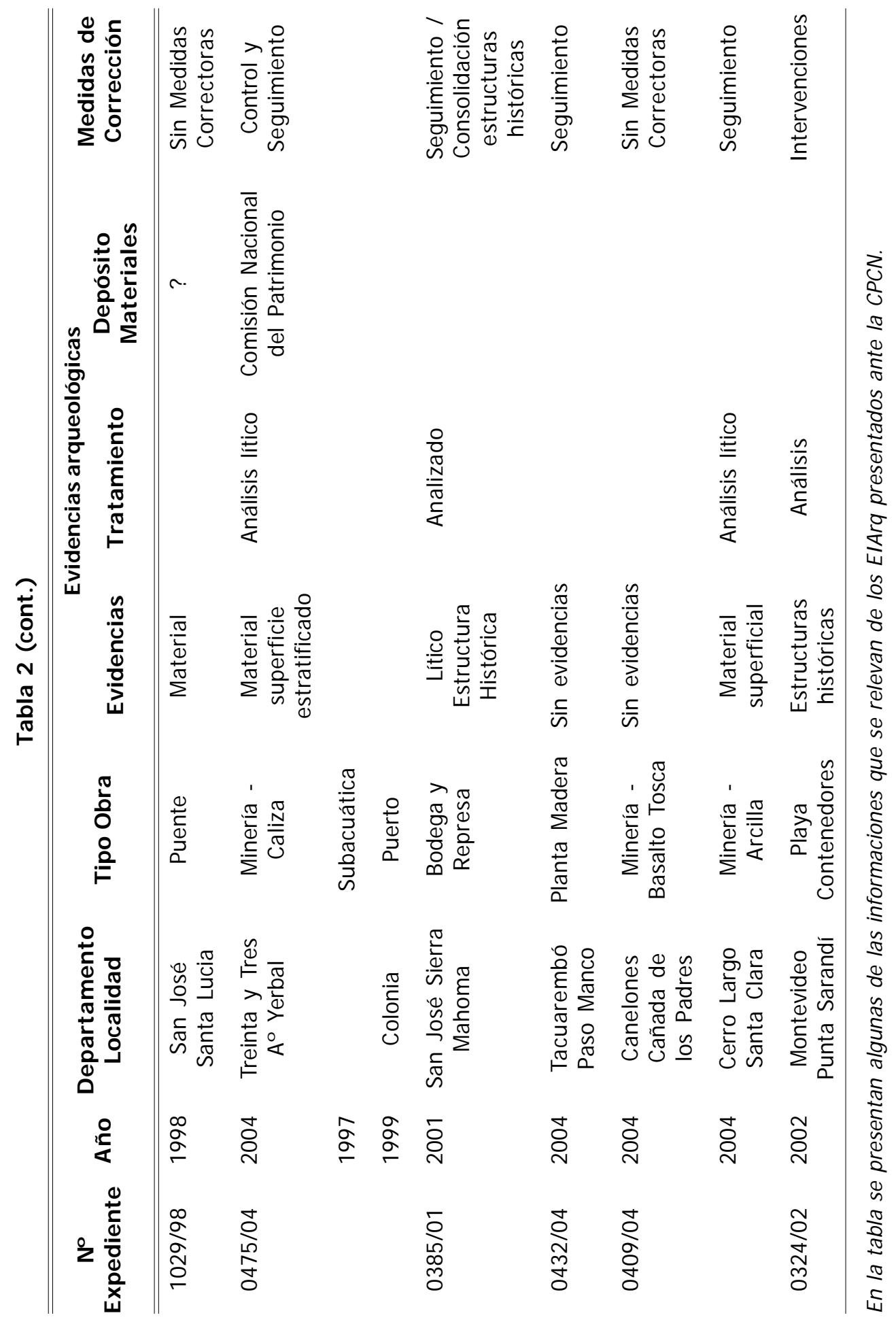


cuenta con planes de Gestión Integral del Patrimonio Arqueológico, el modelo de procesos de trabajo que se presenta se basa en la propuesta realizada por el grupo del Laboratorio de Arqueoloxía da Paisaxe del Instituto de Estudos Galegos Padre Sarmiento (CSIC - Xunta de Galicia). Esta propuesta se considera como un instrumento base para comenzar a lograr una efectiva y dinámica gestión del patrimonio. La propuesta se base en que todo programa de investigación, incluidos los El Arq, debe abordar, en todas sus fases, los diferentes procesos de trabajo: catalogación, valoración, intervención y puesta en valor (Criado 1996a ).

Se considera que sin una gestión efectiva y eficaz los trabajos de ElArq seguirán sin cumplirse con los requisitos mínimos que involucra un estudio de estas características y se continuará con el libertinaje en la actividad arqueológica; perjudicial en primera instancia para el Patrimonio. Las fases de trabajo que se presentan de manera esquemática (ver Amado et al. 2002, Barreiro 2002, Cacheda 2004, Criado et al. 2004, entre otros) intenta contribuir a la gestión de los ElArq y apuesta a un cambio en el accionar de los profesionales involucrados en los mismos (Tabla 3).

\section{Fase de Estudio y Evaluación}

Se entiende que esta fase de estudio y evaluación del impacto que producirán diversos emprendimientos debe considerar los siguientes puntos:

- El objetivo principal en esta fase debería ser diagnosticar, prevenir, mitigar, corregir y/o compensar el impacto, revirtiendo los efectos negativos mediante la producción de conocimiento.

- Realización de una Oferta de Trabajo- Para ello es necesario conocer los bienes culturales del área, la documentación técnica del proyecto de obra, su localización, extensión y conocer los plazos con los que se cuenta. En función de esa información, se plantea el trabajo, el equipo de personas, el período de realización y el presupuesto del mismo.

- Generación de un Proyecto Arqueológico - Por un lado, debería incluirse en el Proyecto una ficha técnica en la que se especifique el tipo de actuación a realizar por el emprendimiento, tipo de actuación arqueológica, zona geográfica de la actuación, equipo técnico de la actuación, periodo de realización, empresa contratante y presupuesto de la misma. Por otro, se resumen los objetivos del proyecto, se realiza la descripción geográfica de la zona de trabajo, se plantea la problemática patrimonial de acuerdo al relevamiento de antecedentes, la metodología y el plan de trabajo a llevar a cabo. Asimismo, se presenta el equipo técnico participante.

- Estudio Arqueológico - Localización de sitios conocidos y sin conocer, registrados por medio de prospecciones (prospección directa superficial extensiva e intensiva y prospección directa subsuperficial), fichas de registro y fotografías que posteriormente serán sistematizadas y analizadas al igual que la cultura material relevada.

- Valoración del impacto - Se realizar un diagnostico de los impactos que el proyecto genera sobre el patrimonio de la zona. Impacto existe cuando hay una afección dentro del entorno de un sitio u área de interés patrimonial. Para determinar el entorno de afección, hay que diagnosticar el tipo de impacto a generarse.

- Plan de corrección - Según el diagnostico de impacto se proponen las medidas correctoras a llevar a cabo.

\section{Fase de Corrección}

En fase de corrección de impacto debe considerarse:

- Entre los objetivos se encuentran: controlar que se adopten las medidas correctoras especificadas en la fase anterior; realizar la inspección de la obra y las remociones de tierra para documentar la aparición de entidades arqueológicas no previstas y poner en practica las acciones previstas en fase de 
Tabla 3

\begin{tabular}{|c|c|c|c|}
\hline Etapa & Finalidad & Actuación & $\begin{array}{c}\text { Fase } \\
\text { Proyectode obra }\end{array}$ \\
\hline \multirow[t]{2}{*}{ EVALUACIÓN } & $\begin{array}{l}\text { Identificar los elementos } \\
\text { patrimoniales y su entorno } \\
\text { de protección. } \\
\text { - Descubrimiento } \\
\text { - Localización } \\
\text { - Descripción } \\
\text { - Documentación } \\
\text { - Estudio } \\
\text { - Valoración } \\
\text { - Difusión de los bienes } \\
\text { patrimoniales }\end{array}$ & $\begin{array}{l}\text { Identificación de los } \\
\text { elementos patrimoniales } \\
\text { - Estudio Cartográfico } \\
\text { - Consultas Bibliográficas } \\
\text { - Prospecciones intensivas y } \\
\text { extensivas Identificar en } \\
\text { proyecto de obra: } \\
\text { - Agentes (infraestructuras, } \\
\text { instalaciones, etc) } \\
\text { - Acción (actividades } \\
\text { concretas que generan el } \\
\text { impacto) } \\
\text { - Afección (modificaciones del } \\
\text { medio físico - destrucción- } \\
\text { alteración-distorsión) } \\
\text { - Momento del impacto } \\
\text { (identificar fase de proyecto } \\
\text { en donde el impacto se } \\
\text { hará efectivo) }\end{array}$ & $\begin{array}{l}\text { Previas al proyecto } \\
\text { definitivo o en fase } \\
\text { de ejecución }\end{array}$ \\
\hline & \multicolumn{2}{|c|}{$\begin{array}{l}\text { Diagnosticar el impacto } \\
\text { - Crítico - desaparición total o parcial } \\
\text { - Severo - grado menor de gravedad } \\
\text { - Moderado - riesgo elativo (visual) } \\
\text { - Compatible - inexistencia de riesgo }\end{array}$} & \\
\hline \multirow{3}{*}{ CORRECCIÓN } & $\begin{array}{l}\text { Evitar o prevenir la } \\
\text { alteración o destrucción, } \\
\text { así como proteger los } \\
\text { bienes mediante mediadas } \\
\text { cauterlares efectivas y/o } \\
\text { preventivas. }\end{array}$ & $\begin{array}{l}\text { Medidas } \\
\text { Preventivas / Protectoras } \\
\text { - Señalizaciones } \\
\text { - Protección física puntual } \\
\text { - Prospecciones } \\
\text { - Sondeos } \\
\text { - Estrategias de control o } \\
\text { vigilancia }\end{array}$ & \multirow{2}{*}{$\begin{array}{l}\text { Previas al proyecto } \\
\text { definitivo o en fase } \\
\text { de ejecución }\end{array}$} \\
\hline & $\begin{array}{l}\text { Minimizar o Compatibilizar } \\
\text { el impacto generando } \\
\text { conocimiento a partir de } \\
\text { la afección }\end{array}$ & $\begin{array}{l}\text { Medidas Paliativas } \\
\text { - Seguimiento arqueológico } \\
\text { - Traslado o reubicación } \\
\text { - Intervenciones puntuales } \\
\text { - Cambio en técnicas } \\
\text { constructivas }\end{array}$ & \\
\hline & $\begin{array}{l}\text { Compensar el impacto } \\
\text { producido mediante un } \\
\text { valor agregado (plus de } \\
\text { conocimiento). }\end{array}$ & $\begin{array}{l}\text { Medidas Compensatorias } \\
\text { - Excavaciones }\end{array}$ & $\begin{array}{l}\text { Fase de ejecución } \\
\text { fase posterior }\end{array}$ \\
\hline
\end{tabular}

Modelo de fases de trabajo para el desarrollo de EIArq. 
estudio y evaluación para corregir, paliar, mitigar o compensar en el impacto.

- Esta fase también incluye una oferta y un proyecto arqueológico de control y seguimiento de obra.

- Se llevan a cabo nuevas tareas de campo en la cual se constata que el proyecto de obra se corresponda con el proyecto evaluado y se realizan nuevas actividades de prospección en las que se pueden detectar nuevas incidencias sobre bienes culturales.

- Se hacen visitas periódicas e informes puntuales. En la fase de control y seguimiento se inspeccionan los nuevos perfiles expuestos, las escombreras y el entrono de los elementos ya documentados en las zonas de riesgo 0 potenciales.

- El informe final generado es el valorativo, en el que se incluyen las fichas de inventario arqueológico, la ubicación del depósito de los materiales y se produce la Memoria técnica final del Proyecto General.

\section{Algunas reflexiones}

Primeramente considerar que el intento por contribuir a establecer al menos algunos criterios, metodologías y sistemas de registro de la información, así como contribuir al inventario patrimonial, es uno de los caminos que algunos arqueólogos hemos estamos intentando generar y seguir. Se considera que ello contribuiría a la consolidación de la actividad arqueológica necesaria para llevar adelante una política que promueva el crecimiento económico y valore la generación de conocimiento sobre el pasado.

Con ello se esta pretendiendo cooperar en la resolución de la controvertida relación entre dos tipos de intereses (progreso socioeconómico y bienes cultura) que involucran diversos actores. Es por ello que se hace entonces necesaria la participación, el diálogo y el planteo de alternativas, por parte de la administración, propietarios, arqueólogos, consultores y empresarios, entre otros. Se hace necesario que entre esas alternativas se consideren las metodológicas relaciona- das con la gestión del impacto sobre el patrimonio cultural, con la finalidad de contribuir a una normalización de la actividad arqueológica. La gestión idónea del Patrimonio debería ser aquella que estableciese al menos criterios fundamentales para poder decidir: que es lo que puede ser preservado (entendiéndose ello en una situación extrema, convengamos que no se puede parar la economía y desarrollo de un país para preservar todo lo que es evidencia cultural, por ello la necesidad de un inventario que permita valorar los bienes); cuáles son las áreas que deberían ser intervenidas por medio de excavaciones arqueológica; ponerse de acuerdo en que se entiende por sitio o yacimiento arqueológico (si una pieza aislada, varias piezas dispersas, agregaciones parcialmente discretas de piezas, etc); hasta que punto la identificación del patrimonio arqueológico significativo podría alterar o impedir la implementación de un emprendimiento económico, entre varios temas más.

Considerar las dos etapas de trabajo expuestas anteriormente como requisitos mínimos para abordar estos tipos de estudios, podría ser la base para la creación de un programa que articule y consensue, los intereses implicados comprendiendo todas las situaciones eventualmente pasibles de registro y ulterior análisis. Asimismo, pueden ser tomadas para llegar a un consenso respecto a las medias de mitigación, rescate 0 interdicción de a cuerdo a los hallazgos oportunamente efectuados. De esta forma no solo se lograría un consenso entre las partes intervinientes sino que le proporcionaría a la CPCN un mecanismo expeditivo e imparcial para expedirse en tiempo y forma respecto a los informes y a la actuación de los profesionales involucrados en los EIArq.

Dada la situación con la que nos encontramos en Uruguay, merece intentar articular planes de gestión del patrimonio que incluyan la atención a sus funciones metodológicas y cognitivas, estableciendo criterios y procedimientos para la identificación, caracterización y valoración de los bienes patrimoniales. La tarea de mejorar este es- 
tado de cosas evidentemente excede en algunos puntos las posibilidades de los arqueólogos, ya que también se requiere una toma de posición gubernamental acerca de las políticas a seguir con respecto al Patrimonio Cultural.

Para ir finalizando, por un lado debemos reconocer que no hemos sido capaces de desarrollar nuestra disciplina para adaptarla a las nuevas necesidades, como expresa Criado (1996) transformándola en un saberpara-hacer cosas que se pueda aplicar a la resolución de problemas prácticos e inmediatos. El dialogo entre arqueólogos, la actualización metodológica de los mismos, las modificaciones $\mathrm{y} / 0$ adecuaciones dentro de la legislación nacional y administrativa, así como en el campo científico-académico, mediante la formación de recursos humanos que permita la correcta inserción de la arqueología dentro de las esferas productivas de y para la sociedad podrían comenzar a cambiar las cosas.

Mediante este trabajo se intenta contribuir al desarrollo de una nueva etapa en lo que han sido los El Arq en Uruguay, tratando de combinar la satisfacción de las demandas socioeconómicas con la producción de conocimiento sobre el pasado mediante el desarrollo de criterios, procedimientos e instrumentos para la Gestión del Patrimonio Cultural.

\begin{abstract}
The current economical development in Uruguay has led to transformations which affect the archaeological heritage when carrying out different enterprises. Its increasing number together with the Environmental Impact Law have come up with an intensification of I mpact archaeological surveys. However, the development of this kind of archaeological surveys hasn't been accompanied by the legal, administrative and academic fields. The problems generated by all this made us think about the present situation of archaeology, the methodological and ethic procedures and the role of the institutions which must control this kind of archaeological surveys.
\end{abstract}

Keywords: Archaological Impact, Labor field

\title{
Bibliografía
}

AMADO REINO, X., D. BARREIRO, F. CRIADO Y M. MARTÍNEZ

2002 Especificaciones para una gestión del impacto desde la Arqueología del Paisaje. TAPA 26.

BARREIRO D.

2002 Un modelo de estudio de impacto arqueológico. En I Congreso de ingeniería civil, territorio y medio ambiente, pp 481-491. Madrid.

2005 Arqueología y Sociedad: una propuesta epistemológica y axiológica para una
Arqueología aplicada. Tesis Doctoral. Facultad de Xeografía e Historia. Universidad de Santiago de Compostela, $620 \mathrm{pp}$.

CACHEDA PÉREZ, M.

2004 La gestión del Impacto Arqueológico en los paisajes culturales de montaña. En Comunicaciones del Laboratorio de Arqueoloxía da Paisaxe. II Congreso Internacional de ingeniería civil, territorio y medio ambiente, pp. 3-17, Santiago de Compostela, Galicia. 
CABREJAS DOMÍNGUEZ, E.

2004 La corrección de impacto arqueológico en Galicia. Análisis de experiencias en el Plan Eólico y otras obras de infraestructura. En Comunicaciones del Laboratorio de Arqueoloxía da Paisaxe. II Congreso Internacional de ingeniería civil, territorio y medio ambiente, pp. 33-49, Santiago de Compostela, Galicia.

CRIADO BOADO, F.

1996 La Arqueología del Futuro ¿el Futuro de la Arqueología?. Trabajos de Prehistoria 53 (1): 15-35. Madrid.

1996a Hacia un modelo integrado de investigación y gestión del Patrimonio Histórico: la cadena interpretativa como propuesta. PH. Boletín Andaluz de Patrimonio Histórico, 16:73-78. Junta de Andalucía, Consejería de Cultura.

2003 Bases para una teoría arqueológica de la gestión del patrimonio cultural. Texto inédito en: Curso de especialización en xestión arqueolóxica do patrimonio cultural. Santiago de Compostela, Galicia.

CRIADO BOADO, F., D. BARREIRO MARTÍNEZ Y X. AMADO REINO

2004 Arqueología y Obras Públicas: ¿excepción o normalidad? En Comunicaciones del Laboratorio de Arqueoloxía da Paisaxe. II Congreso Internacional de ingeniería civil, territorio y medio ambiente, pp. 5174, Santiago de Compostela, Galicia.

DIARIO OFICIAL

1971 Ley de Patrimonio 14.040/71. Publicada el 27 octubre de 1971 - № 18667. Montevideo.

1994 Ley de Medio Ambiente 16.464/94. Publicada el 26 enero de 1994 - № 23977. Montevideo.
1994 Decreto Reglamentario 435/94 de la Ley 16.466. Publicado el 4 de octubre de 1994 - № 24/145. Montevideo.

EXPEDIENTE 0557/01

2001 Informe de Estudio de Impacto. Departamento de Arqueología de la Comisión del Patrimonio Cultural de la Nación MEC.

EXPEDIENTE 0742/02

2002 Informe Estudio de Impacto. Departamento de Arqueología de la Comisión del Patrimonio Cultural de la Nación - MEC.

EXPEDIENTE 0609/05

2005 Informe Estudio de Impacto. Departamento de Arqueología de la Comisión del Patrimonio Cultural de la Nación MEC.

EXPEDIENTE 0122/06

2006 Informe Estudio de Impacto. Departamento de Arqueología de la Comisión del Patrimonio Cultural de la Nación - MEC.

EXPEDIENTE 0123/06

2006 Informe Estudio de Impacto. Departamento de Arqueología de la Comisión del Patrimonio Cultural de la Nación - MEC.

REGISTRO DE MONUMENTOS HISTÓRICOS NACIONALES

2006 Ministerio de Educación y Cultura Comisión del Patrimonio Cultural de la Nación. http://www. patrimoniouruguay.net/ bienes_protegidos.htm. Actualización: Febrero, 2006.

VERDESIO, G.

2004 La mudable suerte del amerindio en el imaginario uruguayo. En Hacia una arqueología de las arqueologías sudamericanas, pp. 115-150. Universidad de los Andes, Facultad de Ciencias Sociales, CESO, Bogotá, Colombia. 